Pacific Journal of Mathematic 


\title{
PROPERTIES PRESERVED IN SUBDIRECT PRODUCTS
}

\author{
ROGER C. LYNDON
}

1. Introduction. A characterization is obtained of those sentences $S$ of the predicate calculus such that $S$ holds for a subdirect product of general algebraic or relational systems ${ }^{1}$ whenever it holds for each component system. We consider formulas in a first order language equipped with symbols for the operations and relations of the systems under consideration, and, in particular, with a symbol for the identity relation. An atomic formula is one obtained by inserting terms in the argument places of a relation symbol. A positive formula is one that can be built up from atomic formulas by means of conjunction, disjunction, and of universal and existential quantification (but without using negation). A special Horn formula is one of the from $P \supset F$ where $P$ is a positive formula and $F$ is an atomic formula, or any formula obtained from such formulas by conjunction and universal quantification. A sentence is a formula without free variables. As a corollary to our main theorem we obtain the following :

A sentence has the property that it holds for a subdirect product of systems whenever it holds for each component system if and only if it is equivalent to a special Horn sentence.

An example of a special Horn sentence is provided by the condition for an associative ring to be semisimple in the sense of Jacobson [7, Proposition 1, p. 9], which is expressed by the following sentence:

$$
\forall z \cdot[\forall x \forall y \exists u \cdot x z y+u=x z y u \wedge u x z y=x z y u] \supset z=0 .
$$

We admit among subdirect products the subdirect product of an empty set of systems, which, from the definition, proves to be a trivial system with a single element and all relations universal. The sole effect of excluding this trivial case would be to admit in special Horn sentences clauses $\sim P$ along with the clauses $P \supset F$.

A. Horn [6] considered the more general class of all sentences obtained by universal and existential quantification from conjunctions of formulas of the type $P \supset F$ (or $\sim P$ ), where $P$ is a conjunction of atomic formulas and $F$ an atomic formula. Horn showed that all such sentences are preserved under (full) direct products, while C. C. Chang and Anne C. Morel [4] showed that there are sentences preserved under direct product that are not equivalent to any such Horn sentence. The problem of characterizing syntactically those sentences preserved under direct

Received October 22, 1958. Work supported in part under grants from the National Science Foundation

1 This concept is due to Tarski; see [13], [14]. 
product, as well as that of determining under what algebraic processes Horn sentences are preserved, remains open. That the general Horn sentence is not preserved under subdirect product is shown by a simple example: the family of all finite subsets of an infinite set constitutes, in the usual sense, a ring without unity, that is, in which the Horn sentence $\exists x \forall y \cdot x y=y$ fails, although it is a subdirect product of twoelement fields, in which this sentence holds.

The earliest result of the kind under consideration is that of $G$. Birkhoff [3] who showed that those classes of algebras that are closed under formation of direct products, subsystems, and homomorphic images are precisely those classes definable by universally quantified equations. In addition to the work of Horn, Chang, and Morel, properties preserved under direct products have been studied by K. Bing [2], K. Appel [1], and A. I. Taimanov [12], while subdirect products have been studied by A. Malcev [11].

We first proved the result stated above by means of the theory of Natural Inference of G. Gentzen [5]. The proof offered here seems preferable in that it is simpler, despite the fact that it contains a double induction (which could, with some artificiality, be removed), and in that it presupposes less. We have tried to make the present exposition readable as it stands to one familiar with the general ideas; but for various details, in particular, for precise definitions, and for an Interpolation Theorem which plays a central role in the argument, we refer to our earlier papers [9], [10].

2. Preliminaries. Let $L$ be a first order language, with operation symbols $w$ of prescribed ranks $\rho(w)$, and relation symbols $r$ of ranks $\rho(r)$, among which is the symbol $e$ for the identity relation, of rank $\rho(e)=2$. A model $\mathfrak{A}$ for $L$ consists of a set of operations $\mathfrak{A} w$ on a certain non-empty domain $A$, and of relations $\mathfrak{U} r$ on $A$, indexed by the operation symbols $w$ and relation symbols $r$ of $L$, and of corresponding ranks. A relational system is a model such that $\mathfrak{A} e$ is the identity relation on the domain $A$ of $\mathfrak{A}$.

Let $\mathfrak{A}_{i}$, for all $i$ in an index set $I$, be relational systems for a language $L$. The direct product $\mathfrak{A}$ of the $\mathfrak{A}_{i}$ is defined as follows. The domain $A$ of $\mathfrak{A}$ is the Cartesian product of the domains $A_{i}$ of the $\mathfrak{A}_{i}$. For each $i$ in $I$ we denote by $\pi_{i}$ the projection carrying each $a$ in $\mathfrak{A}$ onto its component $\pi_{i} a$ in $\mathfrak{H}_{\mathfrak{i}}$. The operations $\mathfrak{A} w$ of $\mathfrak{A}$ are defined by specifying their components : for each $i$, and $a_{1}, \cdots, a_{\rho(w)}$ in $\mathfrak{H}$,

$$
\pi_{i}\left[\mathfrak{A} w\left(a_{1}, \cdots, a_{\rho(w)}\right)\right]=\mathfrak{N}_{i} w\left(\pi_{i} a_{1}, \cdots, \pi_{i} a_{\rho(w)}\right) ;
$$

the relations $\mathfrak{A} r$ of $\mathfrak{A}$ are defined by taking $\mathfrak{A} r\left(a_{1}, \cdots, a_{\rho(r)}\right)$ to hold, for $a_{1}, \cdots, a_{\rho(r)}$ in $\mathfrak{A}$, if and only if $\mathfrak{A}_{i} r\left(\pi_{i} a_{1}, \cdots, \pi_{i} a_{\rho(r)}\right)$ holds in $\mathfrak{A}_{i}$ for each $i$ in $I$. It must be noted that this last criterion is satisfied by the 
identity relation. A system $\mathfrak{Y}^{\prime}$ is a subdirect product of the systems $\mathfrak{N}_{i}$ if it is a subsystem of the direct product $\mathfrak{A}$ such that, for each $i$ in $I$, the projection $\pi_{i}$ maps the domain $A^{\prime}$ of $\mathfrak{Y}^{\prime}$ onto the domain $A_{i}$ or $\mathfrak{\Re}_{i}$.

The usual criterion for an algegraic system to be isomorphic to a subdirect product of systems from a given collection carries over directly to relational systems, and takes the following form.

CRITERION. A relational system $\mathfrak{A}$ is isomorphic to a subdirect product of systems belonging to a given collection $K$ if and only if there exists a family $\Theta$ of homomorphisms $\theta$ of $\mathfrak{A}$ onto systems $\theta \mathfrak{A}$ in $K$ such that for all $r$ and $a_{1}, \cdots, a_{\rho(r)}$ in $\mathfrak{A},[(\theta \mathfrak{A}) r]\left(\theta a_{1}, \cdots, \theta a_{\rho(r)}\right)$ for all $\theta$ in $\Theta$ implies that $\mathfrak{U} r\left(a_{1}, \cdots, a_{\rho(r)}\right)$ in $\mathfrak{A}$.

Before turning to the main theorem we establish a series of lemmas.

Lemma 1 . Let $F$ be a formula with distinct free variables $x_{1}, \cdots, x_{n}$, and $F^{\prime}$ the result of replacing in $F$ the $x_{i}$ by new and distinct constants (operation symbols of rank zero) $w_{i}$. If $C$ is any formula that does not contain the $w_{i}$, and $C \Rightarrow F^{\prime \prime}$, then $C \Rightarrow \forall x_{1} \cdots x_{n} F$.

Proof. ${ }^{2}$ Let $F$ being to a language $L$ that does not contain the $w_{i}$; then $F^{\prime}$ belongs to the language $L^{\prime}$ obtained from $L$ by adjoining the symbols $w_{i}$. Let $\mu$ be an interpretation of $L$ such that $\mu C=1$, and $\lambda$ an interpretation of $L$ that agrees with $\mu$ except on the variables $x_{1}, \cdots, x_{u}$. We must show that $\lambda F=1$. Extend $\mu$ and $\lambda$ to interpretations $\mu^{\prime}$ and $\lambda^{\prime}$ of $L^{\prime}$ by defining $\mu^{\prime} w_{i}=\lambda^{\prime} w_{i}=\lambda x_{i}$. Since $C$ belongs to $L, \mu^{\prime} C=\mu C=1$. Since $C \Rightarrow F^{\prime}$, and $\mu^{\prime} C=1, \mu^{\prime} F^{\prime}=1$. Since $F^{\prime \prime}$ does not contain the $x_{i}, \lambda^{\prime} F^{\prime}=\mu^{\prime} F^{\prime \prime}$, and $\lambda^{\prime} F^{\prime}=1$. By the construction of $F^{\prime}$ and of $\lambda^{\prime}, \lambda F=\lambda^{\prime} F^{\prime \prime}$, whence $\lambda F=1$.

Let $\mathfrak{A}$ be a model for the language $L$, and $L(A)$ the language obtained from $L$ by adjoining new and distinct constants $w_{a}$ for each element $a$ of the domain $A$ of $\overline{\mathfrak{A}}$. Let $\overline{\mathfrak{A}}$ be the extension of $\mathfrak{A}$ to $L(A)$ defined by setting $\overline{\mathscr{A}} w_{a}=a$ for all $a$ in $\mathfrak{A}$. Let $\mu$ be an ordinal number, and $L_{\mu}$ the language obtained from $L(A)$ by adjoining new and distinct relations symbols $r_{\nu}$ of $\operatorname{rank} \rho\left(r_{\nu}\right)=\rho(r)$, for all $r$ in $L$ and $\nu<\mu$. If $\mathfrak{A}_{\mu}$ is any model for $L_{\mu}$, and $\nu<\mu$, let $\mathfrak{A}_{\mu, \nu}$ be the model for $L$ defined by taking $\mathfrak{A}_{\mu, \nu} w=\mathfrak{A}_{\mu} w$ for all $w$, and $\mathfrak{A}_{\mu, \nu} \boldsymbol{r}=\mathfrak{A}_{\mu} \boldsymbol{r}_{\nu}$ for all $\boldsymbol{r}$.

Let $K$ be an elementary class ${ }^{3}$ of relational systems. We shall say that a model $\mathfrak{A}_{\mu}$ of $L_{\mu}$ has the property (*) if

(1) the restriction of $\mathfrak{A}_{\mu}$ to the language $L(A)$ is an elementary extension of $\overline{\mathfrak{H}}$;

2 For concepts appearing in this paper without definition, see [9], [10].

3 As in [10], we use "elementary class" in the sense of Tarski's "arithmetical class in the wider sense $\left(A C_{\Delta}\right) "$. 
(2) the restriction to $L$ of $\mathfrak{A}_{\mu, \nu}$ is in $K$, for all $\nu<\mu$;

( 3 ) $\mathfrak{A}_{\mu} r \subseteq \mathfrak{A}_{\mu, \nu} r$ for all $r$ in $L$ and all $\nu<\mu$.

Lemma 2. For $\mu=0$, the model $\mathfrak{A}_{0}=\overline{\mathcal{U}}$ of the language $L_{0}=L(A)$ has the property $\left({ }^{*}\right)$.

Proof. Condition (1) is trivial, and (2) and (3) are vacuous.

Let $\Sigma$ be the class of all special Horn sentences that hold for $K$, and $\Sigma^{*}$ the class of those models that satisfy all sentences in $\Sigma$.

Lemma 3. Let $\mathfrak{A}$ be in $\Sigma^{*}$, and $F$ an atomic sentence, that is, an atomic formula without free variables, of $L(A)$ that fails in $\overline{\mathfrak{A}}$. Let $\mathfrak{A}_{\mu}$ be a model for $L_{\mu}$ with property $\left(^{*}\right)$. Then there exists a model $\mathfrak{A}_{\mu+1}$ for $L_{\mu+1}$ with property $\left({ }^{*}\right)$ such that

and

(1') the restriction of $\mathfrak{A}_{\mu+1}$ to $L_{\mu}$ is an elementary extension of $\mathfrak{A}_{\mu}$;

(4) $\quad F$ fails in $\mathfrak{H}_{\mu+1}$.

Proof. Let $I$ be the set of all sentences of $L$ that hold in $K$. Let $\Delta$ be the set of all sentences of the language $L_{\mu}\left(A_{\mu}\right)$ that hold in $\overline{\mathfrak{A}}_{\mu}$. Let $\Gamma^{\prime}$ result from $\Gamma$ and $F^{\prime}$ from $F$ by replacing each $r$ by the corresponding $r_{\mu}$. Let $I$ be the set of all sentences

$$
I\left(r, r_{\mu}\right)=\forall x_{1} \cdots x_{\rho(r)} \cdot r\left(x_{1}, \cdots, x_{\rho(r)}\right) \supset r_{\mu}\left(x_{1}, \cdots, x_{\rho(r)}\right),
$$

for all $r$ in $L$.

Suppose the set $\triangle, I, \Gamma^{\prime \prime}, \sim F^{\prime \prime}$ is inconsistent. By the Compactness Theorem, there exists a conjunction of sentences from $\Gamma$, and hence a single sentence $C$ from $\Gamma$, such that $\Delta, I, C^{\prime}, \sim F^{\prime \prime}$ is inconsistent. Thus $\Delta, I \Rightarrow C^{\prime} \supset F^{\prime}$, where $C^{\prime} \supset F^{\prime}$ contains only the relation symbols $r_{\mu}$, while $\Delta$ does not contain the $r_{\mu}$, and $I$ contains the $r_{\mu}$ only positively. By the Interpolation Theorem of [9], there exists a positive sentence $P^{\prime}$ containing only the $r_{\mu}$ such that $\Delta, I \Rightarrow P^{\prime}$ and $P^{\prime} \Rightarrow C^{\prime} \supset F^{\prime}$. If $P$ is the result of replacing each $r_{\mu}$ in $P^{\prime}$ by the corresponding $r$, it follows that $\Delta \Rightarrow P$ and $P \Rightarrow C \supset F$. Thus $C \Rightarrow P \supset F$. Let $P_{0}$ and $F_{0}$ result from $P$ and $F$ by replacing all $w_{b_{i}}$ that occur in them by distinct variables $x_{1}, \cdots x_{n}$. Since $C$ is in $\Gamma$, and belongs to the language $L$ that does not contain the $w_{b}$, it follows by Lemma 1 that $C \Rightarrow H$, where $H=$ $\forall x_{1} \cdots x_{n} \cdot P_{0} \supset F_{0}$. Since $H$ contains only the relation symbols $r$, and does not contain the $w_{b}$, it belongs to the language $L$. Since $H$ is a special Horn sentence, and a consequent of $C$ in $\Gamma, H$ is in $\Sigma$. Since $\mathfrak{A}$ is in $\Sigma^{*}, H$ holds in $\overline{\mathfrak{A}}$, and hence in $\mathfrak{A}$. It follows that $P \supset F$ holds in $\overline{\mathfrak{A}}$. On the other hand, from $\Delta \Rightarrow P$ we have that $P$ holds in $\overline{\mathfrak{A}}_{\mu}$, hence in $\overline{\mathfrak{A}}$. From the fact that $P$ and $P \supset F$ both hold in $\overline{\mathfrak{A}}$ it follows that $F$ holds in $\overline{\mathfrak{A}}$ which contradicts the hypothesis of the lemma. 
It has been established that the set $\Delta, I, \Gamma^{\prime}, \sim F^{\prime \prime}$ is consistent, and therefore holds in some model $\mathfrak{B}$. Let $\sqrt{ }$ be the restriction of $\mathfrak{B}$ to the language $L$. From the fact that $\mathfrak{B}$ satisfies $\Delta$, it follows by Proposition 3 of [10] that the quotient model $\mathfrak{A}_{\mu+1}=\mathbb{E} / \mathfrak{E} e$ is a relational system and an elementary extension of $\mathfrak{A}_{\mu}$. This establishes (1'), and, by virtue of the hypothesis that $\mathfrak{A}_{\mu}$ has the property $\left(^{*}\right)$, it follows that $\mathfrak{A}_{\mu+1}$ satisfies (1) and also (2) and (3) for all $\nu<\mu$. From the fact that $\mathfrak{B}$, and therefore $\mathfrak{A}_{\mu+1}$, satisfies $\Gamma$, it follows that the restriction to $L$ of $\mathfrak{A}_{\mu+1},{ }_{\mu}$ is in $K$, which completes the proof of (2). From the fact that $\mathfrak{B}$, and therefore $\mathfrak{A}_{\mu+1}$, satisfies $I$, it follows that, for all $r, \mathfrak{A}_{\mu+1} r \subseteq \mathfrak{A}_{\mu+1, \mu} r$, which completes the proof of (3). Finally, from the fact that $\mathfrak{B}$ satisfies $\sim F^{\prime \prime}$ it follows that $F^{\prime \prime}$ fails in $\mathfrak{A}_{\mu+1}$, as required by (4).

LEMma 4. Let $\mu$ be a limit ordinal, and a family of systems $\mathfrak{U}_{\nu}$ for $L_{\nu}$, all $\nu<\mu$, be given, with the property $\left({ }^{*}\right)$ and such that

$(1: \mu)$ for all $\rho<\nu<\mu$, the restriction of $\mathfrak{A}_{\nu}$ to $L_{\rho}$ is an elementary extension of $\mathfrak{A}_{\rho}$.

Let $F_{\nu}$, all $\nu<\mu$, be a set of atomic sentences of $L(A)$ such that

$(4: \mu)$ for all $\nu+1<\mu, F_{\nu}$ fails in $\mathfrak{A}_{\mu, \nu+1}$.

Then there exists a model $\mathfrak{A}_{\mu}$ for $L_{\mu}$ with property $\left(^{*}\right)$ and such that $(1: \mu+1)$ and $(4: \mu+1)$ hold.

Proof. By virtue of $(1: \mu)$, the $\mathfrak{A}_{\nu}, \nu<\mu$ constitute an ascending chain of systems and their union is a well defined system $\mathfrak{A}_{\mu}$. Let $\rho<\mu$, and for all $\nu, \rho<\nu \leqq \mu$, let $\mathfrak{B}_{\nu}$ be the restriction of $\mathfrak{U}_{\nu}$ to $L_{\rho}$. Then $\mathfrak{B}_{\mu}$ is the union of the ascending chain of systems $\mathfrak{B}_{\nu}, \rho<\nu<\mu$. Since each $\mathfrak{B}, \rho<\nu<\mu$, is by $(1: \mu)$ an elementary extension of $\mathfrak{A}_{\rho}$, it follows directly from the definition of elementary extension that $\mathfrak{B}_{\mu}$ is an elementary extension of $\mathfrak{A}_{\rho}^{4}$. This suffices to extend $(1: \mu)$ to $(1: \mu+1)$. That $\mathfrak{U}_{\mu}$ has property $\left(^{*}\right)$ follows from this directly. It remains only to note that, since $\mu$ is a limit ordinal, $(4: \mu+1)$ is in fact equivalent to $(4: \mu)$.

Lemma 5. Let $\mathfrak{A}$ be in $\Sigma^{*}$. Then there exists an ordinal $\mu$ and a model $\mathfrak{A}_{\mu}$ for $L_{\mu}$, with property $\left(^{*}\right)$ and such that

$\left(4^{*}\right)$ if any atomic sentence $F$ of $L(A)$ fails in $\overline{\mathfrak{H}}$, then it fails in some $\mathfrak{A}_{\mu, \nu}, \nu<\mu$.

Proof. Let the $F_{\nu}$, all $\nu<\mu$, for some $\mu$, be the set of all atomic sentences of $L(A)$ that fail in $\overline{\mathfrak{A}}$. Let $\mathfrak{A}_{0}=\overline{\mathfrak{A}}$ as in Lemma 2. For some $\nu, \nu<\mu$, suppose that systems $\mathfrak{A}_{\rho}$ have been constructed for all $\rho<\nu$ with property $\left(^{*}\right)$ and satisfying $(1: \nu),(4: \nu)$. If $\nu$ is not a

${ }^{4}$ See Theorem 1.9 of [15]. 
limit ordinal, Lemma 3 with $F=F_{\nu}$ and $\nu-1$ for $\mu$ assures us of $\mathfrak{A}_{\text {, }}$ with the required properties. If $\nu$ is a limit ordinal, Lemma 4 yields the same result. Thus transfinite induction yields a chain of $\mathfrak{A}_{2}$, all $\nu \geqq \mu$. The condition $(4: \mu)$ now gives $\left(4^{*}\right)$.

Lemma 6. Let $\mathfrak{A}$ be in $\Sigma^{*}$. Then there exists an oridinal $\sigma$ and a system $\mathfrak{H}_{\sigma}$ for $L_{\sigma}$ with the property $\left(^{*}\right)$ and such that

$\left(4^{* *}\right)$ if any atomic sentence $F$ of the language $L\left(A_{\sigma}\right)$ fails in $\overline{\mathfrak{A}}_{\sigma}$, then it fails in $\mathfrak{U}_{\sigma, \nu}$ for some $\nu<\sigma$.

Proof. Iteration of Lemma 5 yields a sequence of ordinals $\mu_{0}=0 \leqq \mu_{1} \leqq \mu_{2} \leqq \cdots$ such that $\mathfrak{U}_{0}$ is $\overline{\mathfrak{A}}$; each $\mathfrak{U}_{\mu_{n}}$ has the property $\left(^{*}\right)$; for each $n$, the restriction of $\mathfrak{H}_{\mu_{n+1}}$ to $L_{\mu_{n}}$ is an elementary extention of $\mathfrak{A}_{\mu_{n}}$; and, finally, that if an atomic sentence $F$ of the language

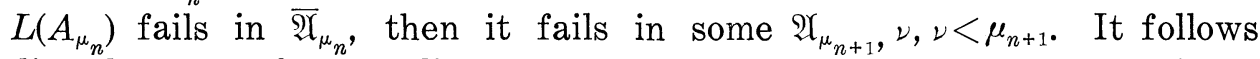
directly that, for $\sigma=\lim _{n<\omega} \mu_{n}$, the union $\mathfrak{A}_{\sigma}$ of the ascending chain of $\mathfrak{V}_{\mu_{n}}, n<\omega$, has the required properties.

\section{The Main Theorem.}

Theorem. Let $\mathfrak{A}$ be a relational system of the language $L$, and $K$ an elementary class of systems of $L$. Then the following are equivalent.

(1) $\mathfrak{U}$ satisfies all special Horn sentences that hold in $K$;

(2) $\mathfrak{U}$ has an elementary extension that is a subdirect product of systems in $K$.

Proof. To show that (2) implies (1), it clearly suffices to show that if $S$ is a sentence of the form $S=\forall x_{1} \cdots x_{n} \cdot P \supset F$ where $P$ is positive and $F$ is atomic, and $\mathfrak{A}$ is a subdirect product of systems $\mathfrak{A}_{\imath}$ in which $S$ holds, then $S$ holds in $\mathfrak{A}$. Suppose then that $S$ holds in all the $\mathfrak{N}_{i}$, and yet $S$ fails in $\mathfrak{A}$. Then there exists an interpretation $\mu$ of $L$ in $\mathfrak{A}$ such that $\mu P=1$ and $\mu F \neq 1$. Since each projection $\pi_{i}$ is a homomorphism of $\mathfrak{A}$ onto $\mathfrak{A}_{i}$, we have that, for all $r$ in $L$ and terms $t_{1}, \cdots, t_{\rho(r)}$, $\mathfrak{U} r\left(\mu t_{1}, \cdots, \mu t_{\rho(r)}\right)$ implies $\mathfrak{U}_{i} r\left(\pi_{i} \mu t_{1}, \cdots, \pi_{i} \mu t_{\rho(r)}\right)$. For each $\mathfrak{A}_{i}$, define an interpretation $\mu_{i}$ in $\mathfrak{A}_{i}$ by setting $\mu_{i} x=\pi_{i} \mu x$. Then $\mu G=1$ implies $\mu_{i} G=1$ for all $i$ if $G$ is an atomic formula, whence $\mu P=1$ implies $\mu_{i} P=1$ for all $i$. Since $S$ holds in each $\mathfrak{H}_{i}$, that $\mu_{i} P=1$ implies $\mu_{i} F=1$, all $i$. But $F$ is an atomic formula, and $\mu_{i} F=1$ implies that $\mu F=1$, a contradiction.

To show that (1) implies (2), assume that $\mathfrak{A}$ is in $\Sigma^{*}$, where $\Sigma$ is the set of all special Horn sentences true for $K$. By Lemma 6 , for some ordinal $\sigma$ there exists a system $\mathfrak{A}_{\sigma}$ of $L_{\sigma}$ with properties $\left(^{*}\right)$ and $\left(4^{*}\right)$. Let $\mathfrak{Y}^{\prime}$ be the restriction of $\mathfrak{U}_{\sigma}$ to the language $L$; by virtue of (1), $\mathfrak{U}^{\prime}$ is an elementary extension of $\mathfrak{A}$. For each $\nu<\sigma$, let $\mathfrak{B}_{\nu}$ be the restric- 
tion of $\mathfrak{\Re}_{\sigma, \nu}$ to the language $L$; by virtue of (2), each $\mathfrak{B}_{\nu}$ is in $K^{* *}$, and consequently the quotient model $\mathfrak{S}_{\nu}=\mathfrak{B} / \mathfrak{B} . e$ is a relational system in $K$. For each $\nu<\sigma, \mathfrak{Y}$ and $\mathfrak{B}$, have the same domain $A$, whence the canonical map $\theta_{\nu}$ of $\mathfrak{A}$ onto $\mathfrak{A} / \mathfrak{B}_{\nu} e$ maps $A$ onto the domain $C_{\nu}$ of $\mathfrak{E}_{\nu}$. By virtue of (3), for each $\nu<\sigma$ and each $r$ in $L, \mathfrak{A T}^{\prime} r=\mathfrak{A}_{\sigma} r \subseteq \mathfrak{A}_{\sigma, \nu} r=\mathfrak{A}_{\nu} r$, whence $\theta_{\nu}$ defines a homomorphism of $\mathfrak{Y}^{\prime}$ onto $\mathfrak{E}_{\nu}$. To complete the proof that the family of $\theta_{\nu}, \nu<\sigma$, satisfies the Criterion for $\mathfrak{X}^{\prime}$ to be a subdirect product of the $\mathbb{F}_{\nu}, \nu<\sigma$, suppose that, for some $r$ in $L$ and $a_{1}, \cdots, a_{\rho(r)}$ in $\mathfrak{A},\left[\mathfrak{夭}_{\nu} r\right]\left(\theta_{2} a_{1}, \cdots, \theta_{2} a_{\rho(r)}\right)$ holds for all $\nu<\sigma$. If $\mathfrak{I}^{\prime} r\left(a_{1}, \cdots, a_{\rho(r)}\right)$ failed in $\mathfrak{U}^{\prime}$, then the atomic sentence $F=r\left(w_{a_{1}}, \cdots, w_{a_{\rho(r)}}\right)$ would fail in $\overline{\mathfrak{A}}$. By virtue of $\left(4^{*}\right), F$ would fail in $\mathfrak{A}_{\sigma, \nu}$, for some $\nu<\sigma$, hence in $\mathfrak{B}_{\nu}$. Since $\mathfrak{B}_{\nu} w_{a_{i}}=\mathfrak{V}_{\sigma} w_{a_{i}}=\mathfrak{A} w_{a_{i}}=a_{i},\left[\mathfrak{B}_{\nu} r\right]\left(a_{1}, \cdots, a_{\rho(r)}\right)$ would fail in $\mathfrak{B}_{\nu}$, whence $\left[\mathfrak{E}_{2} r\right]\left(\theta_{2} a_{1}, \cdots, \theta_{2} a_{\rho(r)}\right)$ would fail in $\mathfrak{F}_{\nu}$. This contradicts our hypothesis, and extablishes the desired conclusion, that $\mathfrak{A l}^{\prime} r\left(a_{1}, \cdots, a_{\rho(r)}\right)$ holds in $\mathfrak{U}^{\prime}$.

4. A complementary example. It will be shown that there exists an elementary class such that the set of all subdirect products of systems from this class is not an elementary class. In consequence, the reference to elementary extensions in the preceding theorem can not be deleted.

If $K$ is any class of systems, let $P(K)$ be the class of all systems isomorphic to some subdirect product of systems from $K$, and let $P_{0}(K)$ be the class of all systems isomorphic to some subdirect product of a non-empty family of systems from $K$. As was noted earlier, $P(K)$ will differ from $P_{0}(K)$ at most in containing all trivial systems, with domain a single element and all relations universal, which will not belong to $P_{0}(K)$ unless $K$ itself contains some trivial system. We suppose now that the language $L$ contains only a finite number of relation symbols, whence there is a single sentence $T$ characterizing the class of ali trivial systems. Then

(1) $P(K)$ is an elementary class if and only if $P_{0}(K)$ is an elementary class.

If $K$ contains a trivial system, then $P(K)=P_{0}(K)$ and there is nothing to prove. Otherwise $P_{0}(K)=P(K)-T$. If $P(K)$ is elementary, say $P(K)=\Gamma^{*}$, then evidently $P_{0}(K)=\{\Gamma, \sim T\}^{*}$ and $P_{0}(K)$ is elementary. On the other hand, if $P_{0}(K)=\Gamma^{*}$, then $P_{0}(K)=\{C \vee T \text { : all } C \text { in } \Gamma\}^{*}$.

If $K$ is any class of systems, let $H^{\prime}(K)$ be the class of all those systems of which some homomorphic image lies in $K$. The following assertion can be obtained by dualizing the proof of the Main Theorem of [10], or may be deduced as a corollary to that theorem.

(2) if $K$ is an elementary class, then $H^{\prime}(K)^{*}$ is the set of all consequences of all negative sentences that hold in $K$. 
It is clear from the definitions that $P_{0}(K) \subseteq H^{\prime}(K)$. To obtain a partial converse, define an occurrence of a relation symbol in a sentence $S$ to be universal if no variable that occurs in the atomic formula containing the given occurrence is existentially quantified in $S$. Then

(3) if $K=\Gamma^{*}$, where no sentence of $\Gamma$ contains a positive universal occurrence of any relation symbol, then $H^{\prime}(K)^{*}=P_{0}(K)^{*}$.

To establish (3), we first show that the argument used to establish the Interpolation Theorem in [9] in fact enables us to impose the following additonal conditions in the conclusion:

(4a) a relation symbol has a positive universal occurrence in $S^{0}$ only if it has a positive universal occurrence in $S$;

(4b) a relation symbol has a positive non-universal occurrence in $S^{0}$ only if it has a positive non-universal occurrence in $T$.

We refer to the proof of the Interpolation Theorem. To prove (4a), suppose that a relation symbol $r$ has no positive universal occurrence in $S=S^{1}$. Then each atomic formula of $S^{1}$ that contains $r$ positively also contains some variable that is existentially quantified in $S^{1}$, whence the corresponding atomic formula in the Skolem matrix for $S^{1}$ contains one of the Skolem functions $s_{i}^{1}$. It follows that each atomic formula in the Skolem matrix $M^{1}$ of $U^{1}$ that contains $r$ positively also contains one of the functions $s_{i}^{1}$, and the same is then true of $M^{0}$, whence it follows that the corresponding atomic formula in $S^{0}$ contains an existentially quantified variable. Since positive occurrences of $r$ in $S^{0}$ can arise only in this fashion, it follows that all such occurrences are non-universal.

To prove $(4 b)$, note first that an atomic formula containing a positive occurrence of $r$ in $S^{0}$ will correspond to an atomic formula $A$ in $M^{0}$ and hence in $M^{1}$, and that, if the occurrence is non-universal, then $A$ will contain one of the functions $s_{0 ;}^{1}$. Suppose now that every positive occurrence of $r$ in $T$ is universal; then in $S^{2}$, equivalent to $\sim T$, we may suppose that every variable that occurs in an atomic formula containing a negative occurrence of $r$ is existentially quantified. Passing to the Skolem matrix of $S^{2}$ and thence to $M^{2}$, it follows that if $B$ is any atomic formula of $M^{2}$ that contains a negative occurrence of $r$, then each occurrence of a variable of $r$ is subordinate to some one of the $s_{0 j}^{2}$ in the sense of occurring in a term beginning with this symbol. From the construction of $M^{0}$ from $M^{1}$ and $M^{2}$ it results that an atomic formula $A$ of $M^{1}$, as above, will appear also in $M^{0}$ only in case $\eta A=\eta B$, for $B$ an atomic formula of $M^{2}$, as described. But this is impossible, since every occurrence of a symbol $s_{v k}^{1}$ in $\eta B$ is subordinate to some $s_{0 i}^{2}$ while $A$ contains an ocurrence of some $s_{0 j}^{1}$ that is not subordinate to any $s_{0 i}^{2}$ in $A$, which does not contain the $s_{0 i}^{2}$, and hence this occurrence of $s_{0 j}^{1}$ is not subordinate to any $s_{0 i}^{2}$ in $\eta A$. 
Turn now to the proof of (3). From the theorem of $\S 3$ it is easy to see that $P_{v}(K)^{*}$ consists of all consequences of 'generalized' special Horn sentences that hold in $K$, that is, of those sentences that hold in $K$ and are obtained by universal quantification and conjunction from formulas of the types $P \supset F$ and $\sim P$, for $P$ positive and $F$ atomic. From the hypothesis of (3), if $\Gamma \Rightarrow T$, where $T$ is a generalized special Horn sentence, then $\Gamma \Rightarrow S$ and $S \Rightarrow T$, where $S$, a conjunction of sentences from $\Gamma$, contains no positive universal occurrence of any relation symbol. Since $T$ contains no positive non-universal occurrences of any relation symbol, application of the Interpolation Theorem with the conditions (4a) and (4b) provides the existence of $S^{0}$ such that $S \Rightarrow S^{0}$ and $S^{0} \Rightarrow T$, where $S^{0}$ contains no positive occurrences of any relation symbol, either universal or non-universal : in short, where $S^{0}$ is negative. Since $\Gamma \Rightarrow S^{0}$ and $S^{0} \Rightarrow T$, it follows by (2) that $T \in H^{\prime}(K)^{*}$. This establishes that $P_{0}(K)^{*} \subseteq H^{\prime}(K)^{*}$, while the opposite inclusion follows from the fact that $P_{0}(K) \cong H^{\prime}(K)$.

In $\S 5$ of [10] an elementary class $K$ of systems, without operations and with a single binary relation (other than identity), was constructed, with the property that $H(K)$ is not elementary. Replacing, in each system in $K$, the relation in question by its complementary relation, yields an elementary class $K^{\prime}$ of systems such that $H^{\prime}\left(K^{\prime}\right)$ is not elementary. More explicitly, $K^{\prime}$ is characterized by the single sentence

$$
S^{\prime}: \quad \exists x y \forall z \exists t: \sim r(x, y) \wedge: \sim r(x, z) \supset \sim r(x, t) \wedge \sim r(z, t)
$$

It follows as in $[10, \S 5]$, or may be derived from the result there, that $H^{\prime}(K)^{*}=\left\{S_{1}^{\prime}, S_{2}^{\prime}, \cdots\right\}^{* *}$, where the $S_{n}^{\prime}$ result from the $S_{n}$ by prefixing a negation $\operatorname{sign}$ to each occurrence of the symbol $r$. If $\mathfrak{U}^{\prime}$ is the natural numbers with the relation $x \leqq y$, it contains descending chains of arbitrary length, hence satisfies the $S_{\imath}^{\prime}$ and belongs to $H^{\prime}\left(K^{\prime}\right)^{* *}$. If $\mathfrak{Y}^{\prime}$ had a homomorphic image $\mathfrak{B}$ in $K^{\prime}$, from $S^{\prime}$ it would follow that $\sim \mathfrak{B} r\left(b_{0}, b_{1}\right), \cdots, \sim \mathfrak{B} r\left(b_{n}, b_{n+1}\right), \cdots$ for some $b_{0}, b_{1}, \cdots$ in $\mathfrak{B}$, and any set of inverse images $a_{v}, a_{1}, \cdots$ would constitute an infinite descending chain in $\mathfrak{Y}^{\prime}$, which is clearly a contradiction. Thus $\mathfrak{Y}^{\prime}$ is not in $H^{\prime}\left(K^{\prime}\right)$, and $H^{\prime}\left(K^{\prime}\right) \neq H^{\prime}(K)^{* *}$, that is, $H^{\prime}\left(K^{\prime}\right)$ is not elementary.

Finally, the set $\Gamma=\left\{S^{\prime}\right\}$ satisfies the hypothesis of (3); indeed, each atomic formula of $S^{\prime}$ contains one of the existentially quantified variables $x, y$ or $t$. Thus, by $(3), P_{0}\left(K^{\prime}\right)^{*}=H^{\prime}\left(K^{\prime}\right)^{*}$. It now follows that $P\left(K^{\prime}\right)$ is not elementary. For, by $(1)$, this would imply that $P_{0}\left(K^{\prime}\right)$ were elementary, hence $P_{0}\left(K^{\prime}\right)^{* *}=P_{0}\left(K^{\prime}\right)$. But $P^{0}\left(K^{\prime}\right)^{*}=H^{\prime}\left(K^{\prime}\right)^{*}$ implies $H^{\prime}\left(K^{\prime}\right)^{* *}=P_{0}\left(K^{\prime}\right)^{* *}$, and $P_{0}\left(K^{\prime}\right) \subseteq H^{\prime}\left(K^{\prime}\right)$, which, together with $P_{0}\left(K^{\prime}\right)^{* *}=$ $P_{0}\left(K^{\prime}\right)$, would imply $H^{\prime}\left(K^{\prime}\right)^{* *} \subseteq H^{\prime}\left(K^{\prime}\right)$ and hence that $H^{\prime}\left(K^{\prime}\right)$ were elementary, a contradiction. 


\section{REFERENCES}

1. K. I. Appel, Horn sentences in identity theory, Notices of Amer. Math. Soc. 6 (1958), 343.

2. K. Bing, On arithmetical classes not closed under direct union, Proc. Amer. Math. Soc. 6 (1955), 836.

3. G. Birkhoff, On the structure of abstract algebras, Proc. Camb. Philos. Soc. 31 (1935), 433.

4. C. C. Chang and Anne C. Morel, On closure under direct product, J. Symbolic Logic 23 (1958), to appear.

5. G. Gentzen, Untersuchungen über das logische Schliessen, Math. Z. 39 (1934-35), 176, 405.

6. A. Horn, On sentences which are true of direct unions of algebras, J. Symbolic Logic 16 (1951), 14.

7. N. Jacobson, The structure of rings, Amer. Math. Soc., Providence, 1956.

8. S. R. Kogalovski, On universal classes of algebras, closed under direct product, Uspehi Mat. Nauk 13 (1958), 241.

9. R. C. Lyndon, An interpolation theorem in the predicate calculus, to Pacific J. Math. $\mathbf{9}$ (1959).

10. R. C. Lyndon, Properties preserved under homomorphism, to Pacific J. Math. 9 (1959). 11. A. I. Malcev, Subdirect products of models, Doklady Akad. Nauk SSSR (N.S.) 109 (1959), 264.

12. A. D. Taimanov, On classes of models, closed under direct product, Uspehi Mat. Nauk 13 (1958), 231.

13. A. Tarski, Some notions and methods on the borderline of algebra and metamathematics, Proc. Internationa Congress of Math. 1, Cambridge, 1950, 705.

14. A. Tarski, Contributions to the theory of models, I, II, III, Indag. Math. 16 (1954), 572,$582 ; 17$ (1955), 55.

15. A. Tarski and R. L. Vaught, Artithmetical extensions of relational systems, Composition Math. 13 (1957), 81.

\section{UNIVERSITY OF MICHIGAN}




\section{PACIFIC JOURNAL OF MATHEMATICS}

\section{EDITORS}

\section{David Gilbarg}

Stanford University

Stanford, California

R. A. Beaumont

University of Washington

Seattle 5 , Washington
A. L. Whiteman

University of Southern California

Los Angeles 7, California

L. J. PAIGE

University of California

Los Angeles 24, California

\section{ASSOCIATE EDITORS}
E. F. BECKENBACH
C. E. BURGESS
E. HEWITT
A. HORN

\author{
V. GANAPATHY IYER \\ R. D. JAMES \\ M. S. KNEBELMAN \\ L. NACHBIN
}
I. NIVEN
E. G. STRAUS
T. G. OSTROM
H. L. ROYDEN
G. SZEKERES
M. M. SCHIFFER
F. WOLF
K. YOSIDA

\section{SUPPORTING INSTITUTIONS}

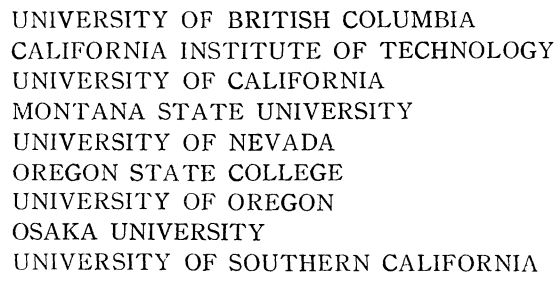

UNIVERSITY OF BRITISH COLUMBIA CALIFORNIA INSTITUTE OF TECHNOLOGY UNIVERSITY OF CALIFORNIA MONTANA STATE UNIVERSITY UNIVERSITY OF NEVADA OREGON STATE COLLEGE UNIVERSITY OF OREGON OSAKA UNIVERSITY UNIVERSITY OF SOUTHERN CALIFORNIA

STANFORD UNIVERSITY

UNIVERSITY OF TOKYO

UNIVERSITY OF UTAH

WASHINGTON STATE COLLEGE

UNIVERSITY OF WASHINGTON

AMERICAN MATHEMATICAL SOCIETY CALIFORNIA RESEARCH CORPORATION HUGHES AIRCRAFT COMPANY SPACE TECHNOLOGY LABORATORIES

Printed in Japan by Kokusai Bunken Insatsusha (International Academic Printing Co., Ltd.), Tokyo, Japan 


\section{Pacific Journal of Mathematics}

\section{Vol. 9, No. 1 \\ May, 1959}

Julius Rubin Blum and Murray Rosenblatt, On the structure of infinitely

divisible distributions . ............................. 1

Robert Geroge Buschman, Asymptotic expressions for

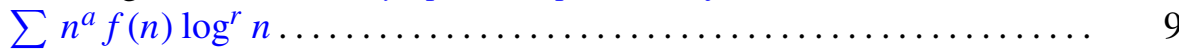

Eckford Cohen, A class of residue systems $(\bmod r)$ and related arithmetical

functions. I. A generalization of Möbius inversion .............. 13

Paul F. Conrad, Non-abelian ordered groups ................... 25

Richard Henry Crowell, On the van Kampen theorem............... 43

Irving Leonard Glicksberg, Convolution semigroups of measures ........ 51

Seymour Goldberg, Linear operators and their conjugates ............ 69

Olof Hanner, Mean play of sums of positional games .............. 81

Erhard Heinz, On one-to-one harmonic mappings ................ 101

John Rolfe Isbell, On finite-dimensional uniform spaces . . ........... 107

Erwin Kreyszig and John Todd, On the radius of univalence of the function

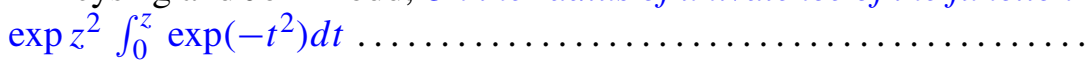

Roger Conant Lyndon, An interpolation theorem in the predicate

calculus......................................... 129

Roger Conant Lyndon, Properties preserved under homomorphism ........ 143

Roger Conant Lyndon, Properties preserved in subdirect products ....... 155

Robert Osserman, A lemma on analytic curves ................ 165

R. S. Phillips, On a theorem due to Sz.-Nagy..................... 169

Richard Scott Pierce, A generalization of atomic Boolean algebras ....... 175

J. B. Roberts, Analytic continuation of meromorphic functions in valued fields................................. 183

Walter Rudin, Idempotent measures on Abelian groups ................ 195

M. Schiffer, Fredholm eigen values of multiply-connected domains ........ 211

V. N. Singh, A note on the computation of Alder's polynomials ......... 271

Maurice Sion, On integration of 1-forms ...................... 277

Elbert A. Walker, Subdirect sums and infinite Abelian groups........... 287

John W. Woll, Homogeneous stochastic processes . .................. 293 